\title{
Activity Modeling for Integrating Environmental, Health and Safety (EHS) Consideration as a New Element in Industrial Chemical Process Design
}

\author{
Hirokazu SugiYama ${ }^{1}$, Masahiko HiraO², \\ Ulrich FISCHER $^{1}$ and Konrad HUNGERBÜHLER ${ }^{1}$ \\ ${ }^{1}$ Institute for Chemical and Bioengineering, ETH Zurich, \\ Wolfgang-Pauli-Strasse 10, 8093 Zurich, Switzerland \\ ${ }^{2}$ Department of Chemical System Engineering, The University of Tokyo, \\ 7-3-1, Hongo, Bunkyo-ku, Tokyo 113-8656, Japan
}

Keywords: Activity Model, Integrated Framework, Environment Health Safety (EHS), Life Cycle Assessment (LCA), Methyl Methacrylate (MMA)

\begin{abstract}
In this paper, we present activity models of chemical process design integrating environmental, health and safety (EHS) evaluation as a new element with conventional economic and technical considerations on the basis of the design framework, which defines different stages of early process design with appropriate indicators for multiobjective evaluation. The type-zero method of Integrated DEFinition language or IDEF0 is selected as an activity modeling method for the hierarchical and transparent description of complex design activities. The viewpoint of the activity model is the user of this design framework, i.e. a design-project manager who leads a group of process chemists and engineers. A set of template models is developed, which describes how a manager executes a project step by step with providing instructions and resources appropriately. According to this template, IDEF0 models of the design framework are created, and here EHS-related activities, tools and information are systematically defined as a part of the whole design activity. As further analysis, sub-activities are presented in detail, where the manager allocates resources appropriately to different design stages, including methods of environmental Life Cycle Assessment (LCA) and EHS hazard evaluation. Another detailed analysis is on how design constraints, e.g., market situation, competitors' patents and company culture, affect different decision-making within the framework. The actual development of methyl methacrylate (MMA) processes is investigated as a case study. The paper finally presents important know-how for the design manager in executing the integrated design framework.
\end{abstract}

\section{Introduction}

Business modeling approach is useful to integrate new mechanisms into existing business process. An analysis of existing activities (often called an "as-is" model), and/or a definition of the work procedure after including them (also called a "to-be" model) helps smoothen the transition of a company's business style into a new one (Naka, 2006). In process industry, several authors have presented applications and merits of such business-modeling techniques, e.g., effective incorporation of individual software tools in the entire life-cycle of process design by Schneider and Marquardt (2002), and the integrated development and management concept by Naka (2006).

The type-zero method of Integrated DEFinition language or IDEF0 (Ross, 1985; National Institute of Standards and Technology, 1993) is a standardized

Received on October 4, 2007; accepted on May 16, 2008. Correspondence concerning this article should be addressed to M. Hirao (E-mail address: hirao@chemsys.t.u-tokyo.ac.jp). method of enterprise-resource planning or businessprocess (re)engineering (Killich et al., 1999). Having originated from Structured Analysis and Design Technique (SADT by Ross, 1977; Ross and Schoman, 1977), IDEF0 was developed through the Integrated Computer Aided Manufacturing (ICAM) program of the U.S. Air Force (1974), aiming at more efficient material supply using computer technologies. In process engineering, different authors have applied this activity modeling technique to the integration of new software tools (Fuchino and Shimada, 2003; Gabber et al., 2004) or non conventional criteria such as environmental or human risks (Fuchino et al., 2004; Sugiyama et al., 2006; Kikuchi and Hirao, 2007) to existing process- or operation-design. There is a project called Process Industries Executive for Achieving Business Advantage Using Standards for Data Exchange (PIEBASE, 2007) where IDEF0 is used to standardize work processes and information requirements within process industries.

As a new philosophy of the chemical industry, sustainable process design is receiving increasing 
Process Chemistry I Process Chemistry II Conceptual Design I Conceptual Design II (PC I) (PC II) (CD I) (CD II)

\begin{tabular}{|c|c|c|c|c|c|}
\hline 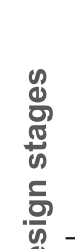 & $\begin{array}{l}\text { Modeled } \\
\text { aspects }\end{array}$ & 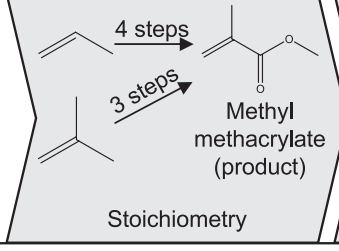 & $\int \underbrace{\longrightarrow}_{\begin{array}{l}\text { Educts, } \\
\text { Conversion/Selectivity; } \\
\text { Temperature/Pressure }\end{array}}$ & $\underset{\text { Shortcut models }}{\stackrel{\perp}{\perp}}$ & $\underbrace{}_{\text {Rigorous models }}$ \\
\hline ดั & $\begin{array}{l}\text { Decision } \\
\text { structure }\end{array}$ & No decision forced & $\begin{array}{l}\text { Select some } \\
\text { reaction route(s) }\end{array}$ & $\begin{array}{l}\text { Select process } \\
\text { option(s) \&/or route(s) }\end{array}$ & Optimize parameters \\
\hline 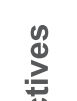 & Economy & $\begin{array}{l}\text { Raw material cost } \\
+\mathrm{MLI}^{1} \text { as proxy }\end{array}$ & $\begin{array}{l}\text { Raw material cost } \\
+\mathrm{ELI}^{3} \text { as proxy }\end{array}$ & Production cost & Net present value \\
\hline $\begin{array}{l}\frac{.}{0} \\
\frac{0}{0} \\
\frac{1}{0}\end{array}$ & $\begin{array}{l}\text { Life-cycle } \\
\text { environmental } \\
\text { impacts }\end{array}$ & $\begin{array}{l}\text { Raw material LCA } \\
+\mathrm{MLI} \text { as proxy }\end{array}$ & $\begin{array}{l}\text { Raw material LCA } \\
+ \text { ELI as proxy }\end{array}$ & Cradle-to-gate LCA & (updated) \\
\hline "̄y & EHS hazard & $\begin{array}{l}\text { ETH-EHS method² } \\
\text { (substance level) }\end{array}$ & $\begin{array}{l}\text { ETH-EHS method } \\
\text { (process level) }\end{array}$ & (updated) & (updated) \\
\hline 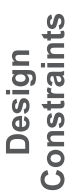 & $\begin{array}{l}\text { Technical } \\
\text { aspects }\end{array}$ & $\begin{array}{l}\text { e.g., market situation; } \\
\text { raw material access; } \\
\text { patent situation; } \\
\text { blacklist substances }\end{array}$ & e.g., catalyst activity & $\begin{array}{l}\text { e.g., } \\
\text { existing know-how; } \\
\text { legislation }\end{array}$ & $\begin{array}{l}\text { e.g., } \\
\text { equipment } \\
\text { specification }\end{array}$ \\
\hline
\end{tabular}

Fig. 1 Overview of framework: definition of design stages and appropriate modeling approaches as well as evaluation indicators attached to each stage; 1, Mass Loss Indices (MLI) by Heinzle et al. (1998); 2, ETH-EHS method by Koller et al. (2000); 3, Energy Loss Index (ELI) by Sugiyama (2007)

attention. Environment, health and safety (EHS) has been the center of the interest of many authors, and many indicators have been proposed to evaluate individual EHS aspects, e.g., the Waste Reduction Algorithm by Hilaly and Sikdar (1995). On top of these methods for assessing individual EHS aspects, several authors have proposed integrated approaches by combining economic and environmental indicators, e.g., Kniel et al. (1996), Azapagic and Clift (1998), CanoRuiz and McRae (1998), Cano-Ruiz (2000), Kheawhom and Hirao (2004a, 2004b). BASF's ecoefficiency analysis (Saling et al., 2002) was introduced as a representative industrial effort that combines various non-monetary issues into a single index compared with economic performance. Different authors have further extended the scope of integration over the time axis: Hoffmann et al. (2001), Chen and Shonnard (2004) and Sugiyama et al. (2008) presented design frameworks that cover different design stages with multiple aspects considered as design objectives. However, many papers have left unclear how a user, who is not an expert in the proposed method, can apply it in industrial process development.
We present an IDEF0 activity model of a multiobjective process design framework (Sugiyama et al., 2008). This novel framework defines four stages of early process design, i.e. Process Chemistry I/II for specifying reaction chemistry and Conceptual Design I/II for selecting process and utility technology. The viewpoint of the activity model is the user of this design framework, i.e. a design-project manager who leads a group of process chemists and engineers. A set of template models is created, which describes how a manager should execute a project step by step while providing appropriate conditions and resources to chemists and engineers. According to this template, IDEF0 models of the design framework are created, and EHS-related activities, tools and information are systematically defined as new elements. As an additional analysis, sub-activities are presented in detail, where the manager allocates appropriate resources to different design stages, including the methods of environmental Life Cycle Assessment (LCA) and EHS hazard evaluation. The actual development of methyl methacrylate (MMA) processes is investigated as a case study. 


\section{Method}

\subsection{Integrated process design framework}

Figure 1 shows an overview of the framework (Sugiyama et al., 2008), which is the target of the activity modeling. It covers the early phase of a grass root design and defines four design stages: Process Chemistry I/II and Conceptual Design I/II. These stages are separated according to the available information for process modeling and to the character of process assessment. As design objectives, the following three aspects are considered: economy, environmental impacts through product's life-cycle, and hazard in terms of EHS. LCA (ISO 14040-43, 1997-2000) and ETHEHS (Koller et al., 2000) methods are selected as appropriate indicators for non-monetary evaluation. As an impact category of LCA, the Cumulative Energy Demand (CED; Verein Deutscher Ingenieure, 1997), which is an energy equivalency of different primary sources used for the production, is selected. At Process Chemistry I/II, proxy indicators are defined to estimate consequential process energy consumption, as a complement to raw material cost/LCA. These quantitative indicators are applied with expanding evaluation scopes, e.g., from substance level to process levels for EHS hazard. In contrast to the above objective functions, technical constraints are considered qualitatively throughout all four stages. A stage-gate approach is taken in this framework: at each stage, reaction and/or process alternatives are modeled and evaluated, and promising one(s) survive(s) to the next design stage. An ideal situation is assumed in applying this framework, i.e. product quality, production scale and location are fixed prior to the first stage, and the handling of the uncertainty is out of the scope within the current framework.

\subsection{Syntax of IDEF0 activity modeling}

The syntax of an IDEF0 model is shown in Figure 2 (a). The box represents function or activity, which has a verb as a name. The input arrows entering the activity box from the left side represent the objects that are transformed by the function into the output arrows on the right side. The control arrows associated with the top side indicate the conditions required to produce the correct output. The mechanism arrows on the bottom indicate the means of performing the function. As shown in Figure 2(b), every activity can be decomposed into sub-level activity models that have the same boundary as the parent activity. Therefore, it is possible to describe complex activities in detail and transparently while having a good overview of the entire activity. The box at the highest level, called "topactivity A0", represents the aggregation of all sub-activities. In this paper, the level directly below top-activity A0 is called "main-level", and activities at the main-level are called "main-level activities". The level where sub-activities of activity $\mathrm{A} i$ are contained is

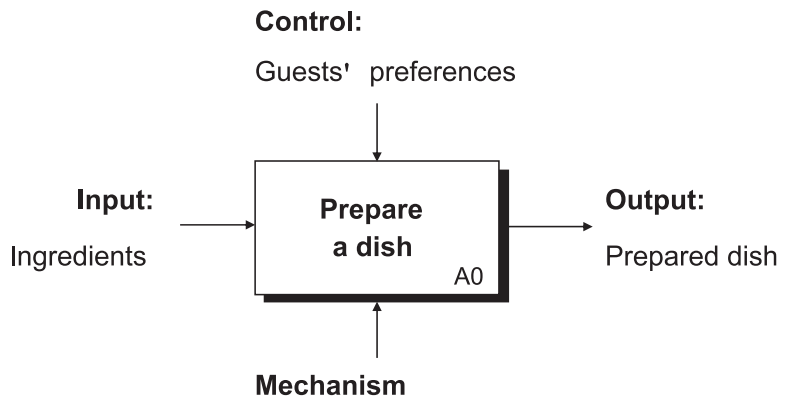

Kitchen facilities, recipe

(a)

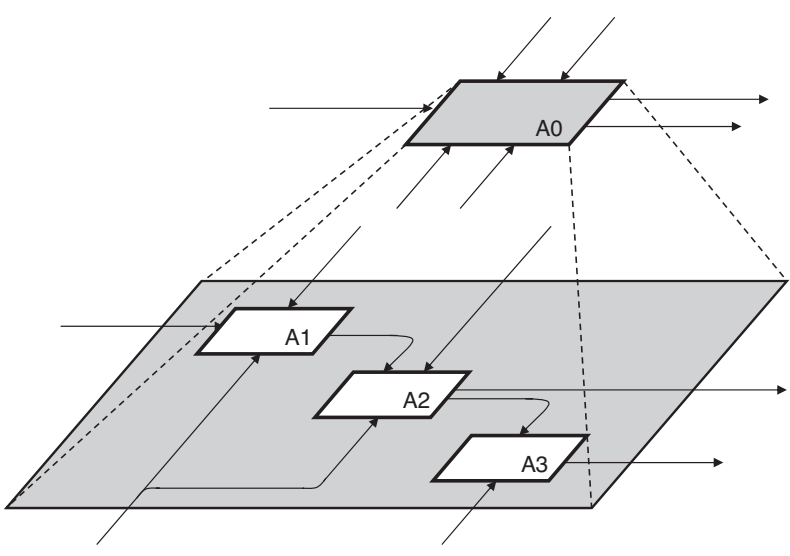

(b)

Fig. 2 (a) Syntax of IDEF0 with example activity: Prepare a dish; (b) decomposition of topactivity A0 to sub-activities. As an example, sub-activities A1 to A3 are defined here. In the same way, each of activities A1 to A3 can be further decomposed to subactivities

called the "A $i$-level", and "A $i$-level activities" refer to these sub-activities.

\subsection{Template for activity modeling "Execute a project"}

In this section, we present a set of IDEF0 models that function as a template for representing the integrated design framework. The top activity of the template is "Execute a project". This model is described from the viewpoint of a project manager who carries out a project while fulfilling different constraints and utilizing available resources.

Figure 3 shows the template of main-level activities. Activity A1: Manage main-level activities is performed by the project manager to control all other activities. It receives input- and control-arrows from the outside of the project, "Input to the project" such as ideas for the project within the company, and "External constraints to the project" such as the capacity of the company. Activity A1 also receives control arrows 


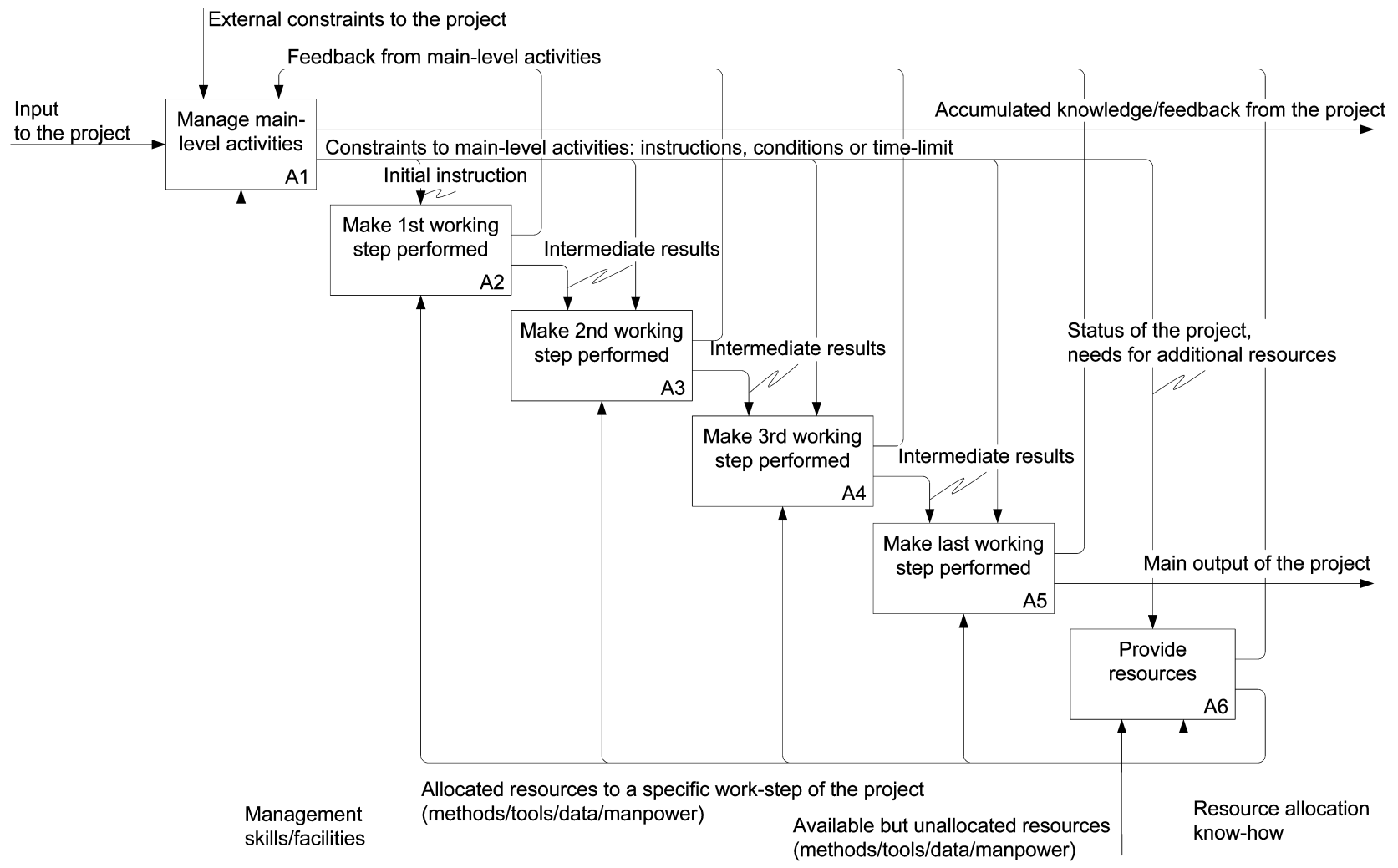

Fig. 3 Template of activity: Execute a project, described from the viewpoint of a project manager; activities A1 and A6 are called management activities, and activities A2 to A5 are referred to as work-step activities

from the activities within the project as "Feedback from main-level activities". On the basis of these inputs and constraints, this activity produces "Constraints to mainlevel activity" such as instructions, conditions or time limits to activities A2 to A6. Another output of activity A1 is "Accumulated knowledge/feedback from the project" as a report that can be communicated with the higher managers or used in other projects. Actual works of the project are performed in activities A2 to A5, which are called work-step activities in this template. In general, the content, character and number of these work-step activities depend on the project. "Initial instruction" is received by activity A2, then "Intermediate results" are produced step by step through activities A3 and A4, and finally "Main output of the project" is obtained in activity A5. The resources for these workstep activities are allocated from activity A6: Provide resources. In this activity, the project manager receives "Status of the project, needs for additional resources" as the control arrow from activity A1, and in response, provides resources required at each work-step activity.

In this template, two types of activities are differentiated. Activities A1 and A6 are defined as management activities, which are executed directly by the project manager. In contrast, work-step activities (Activities A2 to A5) are executed by human resources that the manager assigns from activity A6. The intention of this separation is to highlight the role of the project manager during the entire project time in handling constraints and resources.

The activity model shown in Figure 3 covers different patterns of project execution. In the most ideal case, each of the work-step activities is carried out in the forward direction with appropriate instructions and sufficient resources from management activities. The iteration paths "Feedback from main-level activities" are defined to activity A1 from the other activities. These paths are activated in non-ideal cases, when requests arise from work-step activities for redoing the previous step or for more resources. These requests are handled in activity A1 by the project manager.

Figure 4(a) shows the decomposition of activity A1: Manage main-level activities. Activities A11 and A12 receive project-external inputs and constraints, and project-internal feedbacks, respectively. The project manager decides in activity A13 to which destination extracted information should be directed, i.e. inside or outside of the project. This is a key activity in managing constraints where "Management skills" are fully exploited as a mechanism. Extracted information is summarized in activities A14 and A15, and produced as an output for the specified destination. Between the origin of the received constraints and the destination of the produced output, $2 \times 2$ patterns exist. In the nonideal cases, when the manager decides to solve the 


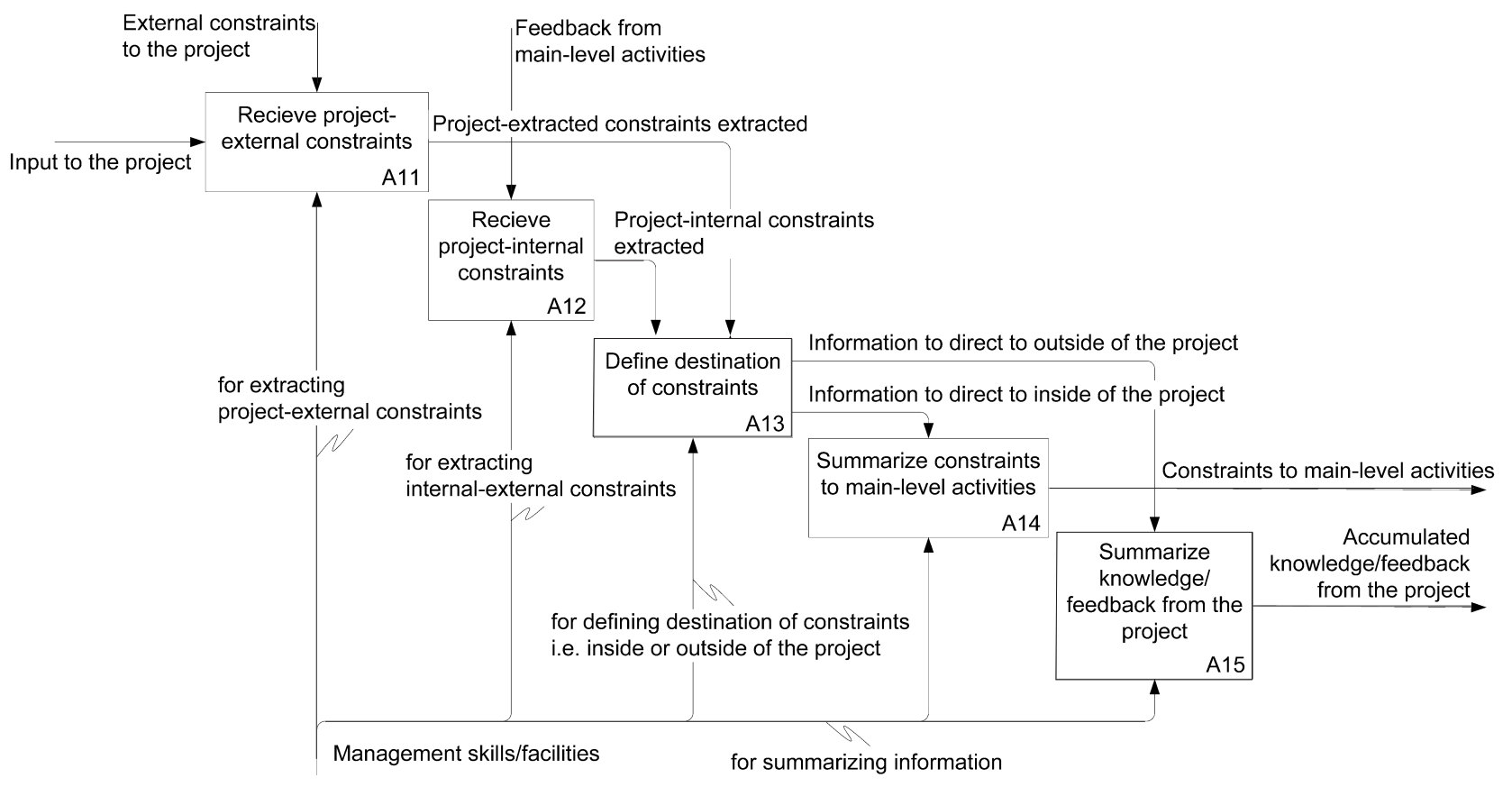

(a)

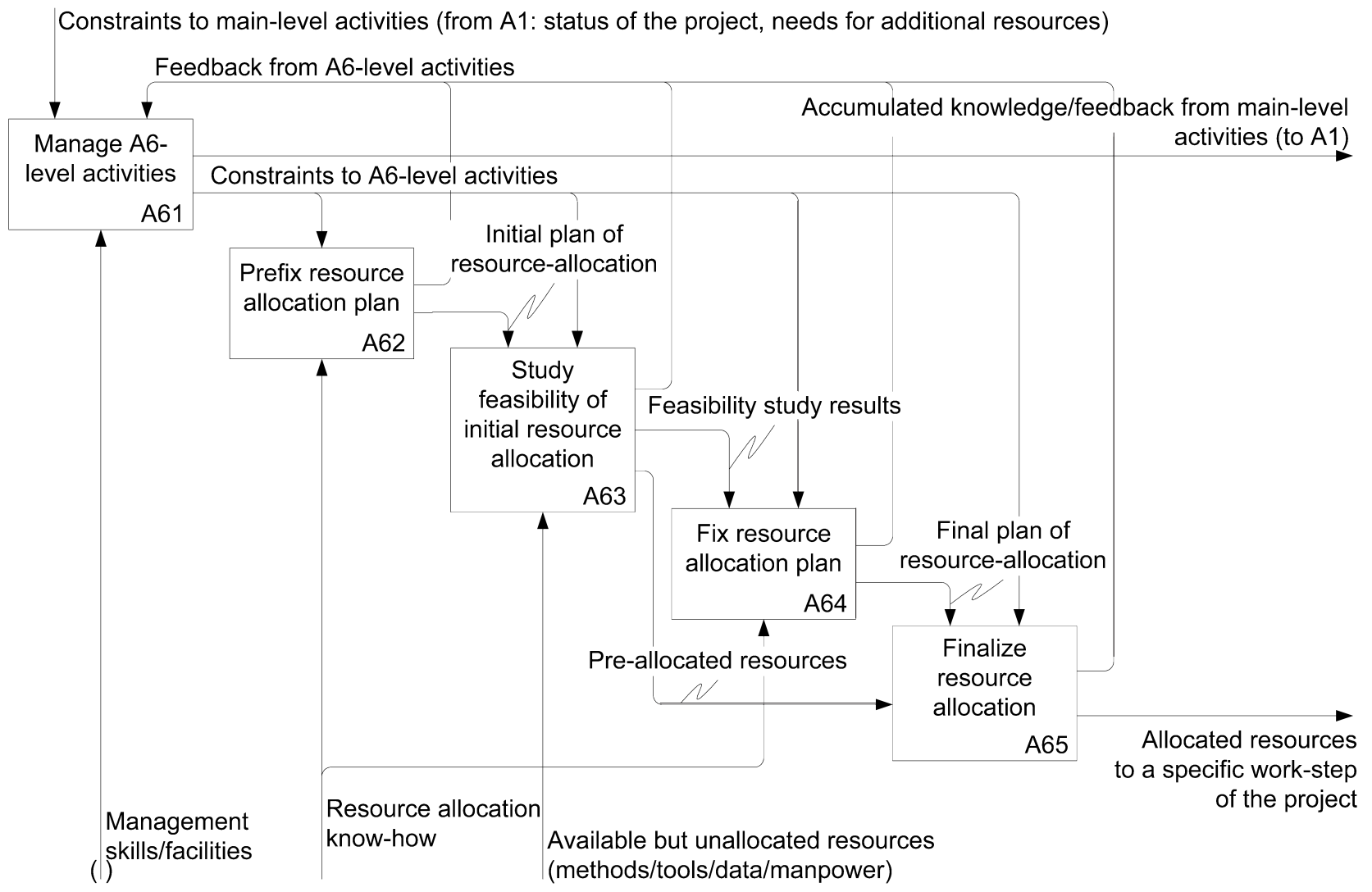

(b)

Fig. 4 Template of sub-activities in activity A1: Manage main-level activities (a) and in activity A6: Provide resources (b) in Figure 3 


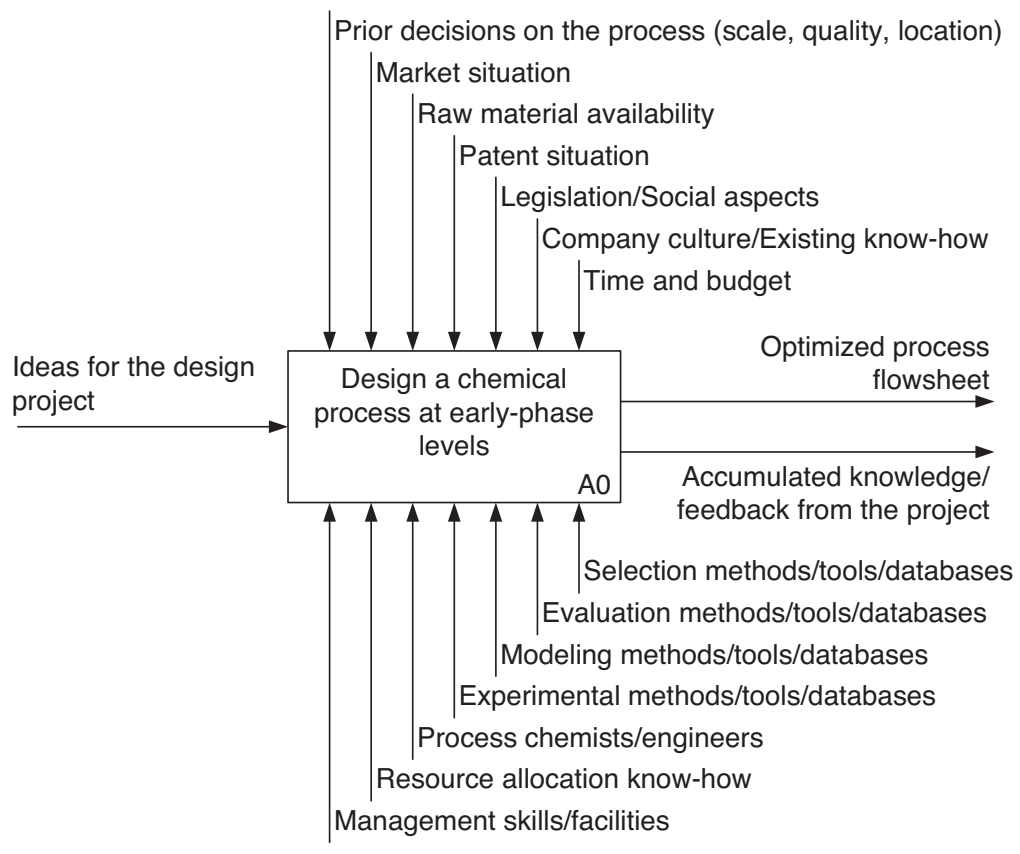

Fig. 5 Top-activity A0: Design a chemical process at early-phase levels

problem inside the project in activity A13, the output is produced in activity A14 to main-level activities; when some external help is judged necessary, the output is produced in activity A15 to outside of the project.

The decomposition of another management activity, A6: Provide resources, is shown in Figure 4(b). In activity A61, the project manager controls all other activities at the same level. This activity receives "Status of the project, needs for additional resources" from activity $\mathrm{A} 1$, and initiates the provision of appropriate resources. In activity A62, the project manager produces an "initial plan of resource allocation". Its feasibility is studied in activity A63, and "feasibility study results" is produced. According to the final plan, "Preallocated resources" from activity A63 are transferred to the final status "Allocated resources to a specific work-step of the project." When the result of the feasibility study is negative, such a non-ideal status is propagated through the feedback loop to activity A61, and further up to activity A1.

\section{Results and Discussion}

\subsection{IDEF0 representation of integrated process design framework}

The IDEF0 representation of integrated design framework is described in this section. The set of templates presented in Section 1.3 is applied. Figure 5 shows the top-activity A0: Design a chemical process at early phase levels. The viewpoint of this model is a design-project manager who carries out a project of process design as shown in Figure 1 under design constraints and with given resources. The overall input is
"Ideas for the design project" that triggers the whole project. There are seven constraints, which correspond to the "External constraints to the project" in the template shown in Figure 3. "Prior decisions on the process" is the necessary constraint of the design regarding production scale, product quality and process location. "Market situation," "Raw material availability," "Patent situation," and "Legislation/Social aspects" are enterprise-exogenic constraints, whereas "Company culture/Existing process" and "Time and budget" are enterprise-endogenic ones. On the side of the mechanism, the "Management skills/facilities" and "Resource allocation know-how" shown in Figure 3 are also defined here. The remaining mechanism arrows correspond to "Available but unallocated resources" in Figure 3, i.e. "Process chemists/engineers," and "Methods/tools/databases" for experiments, modeling, evaluation and selection. Based on these incoming information and resources, the overall outputs "Optimized process flowsheet" and "Accumulated knowledge/feedback from the project" are produced. The former output is a direct input for the successive process development phases, piloting and detailed engineering.

Figure 6 shows the main-level activities or the decomposition of top-activity A0 according to the template shown in Figure 3. Management activities in the template are defined here as activities A1 and A6. Four stages in the framework from Process Chemistry I to Conceptual Design II, as shown Figure 1, are defined as work-step activities. The design-project manager makes individual steps performed by process chemists or engineers that he/she provides in activity A6. In this activity, these project members are trained 


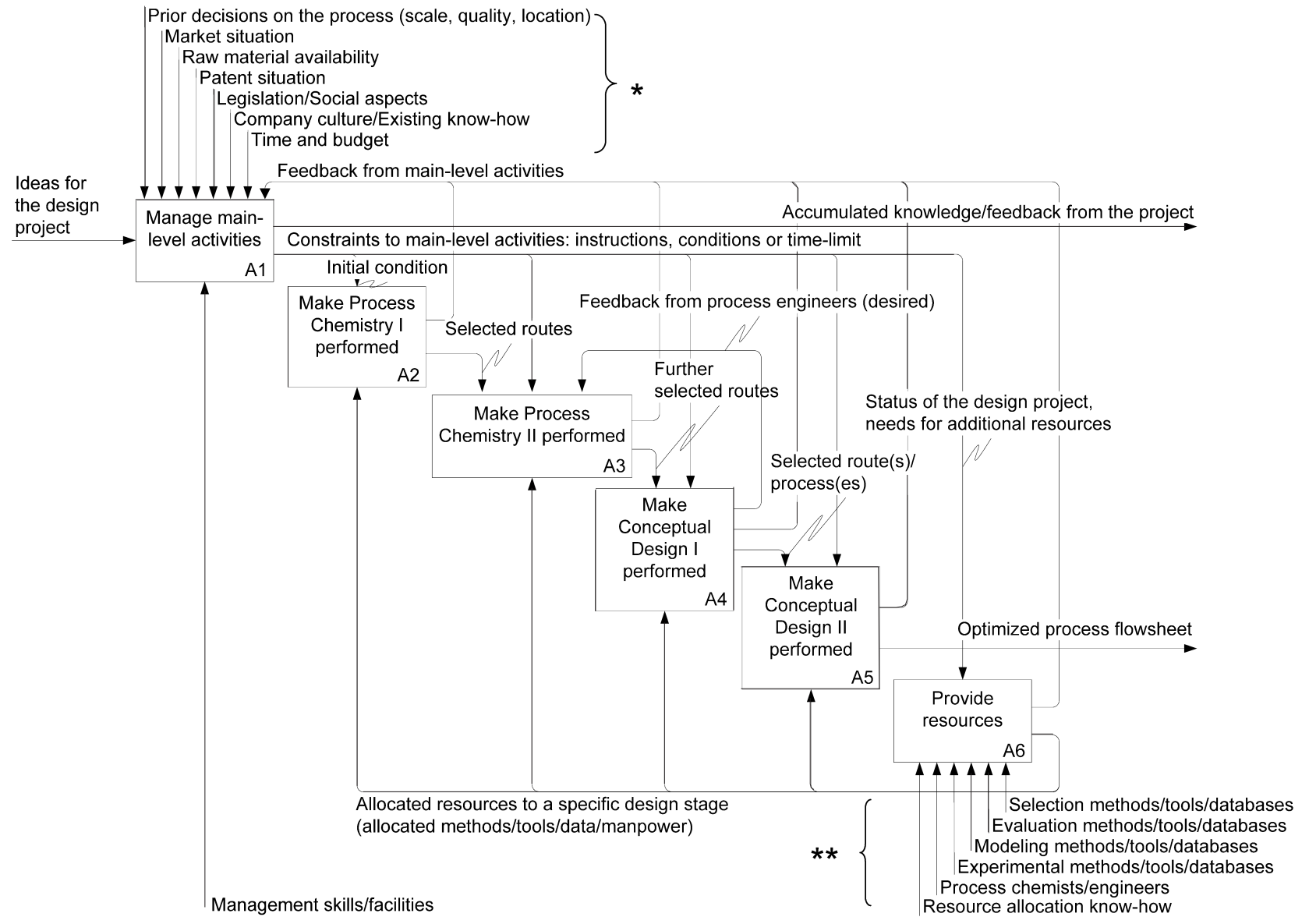

Fig. 6 Main-level of activity: Design a chemical process according to the framework based on the template in Figure 3; $*$ and ** correspond to "External constraints to the project" and "Available but unallocated resources" in Figure 3

to become familiar with methods, data and tools for experiments, modeling and evaluation. Thus, chemists and engineers, when allotted to each design stage, know how to perform LCA and EHS hazard evaluation, which is new to conventional practice. Multiobjective decisions on process alternatives are made step by step in activities A2 to A5, and the final outcome, "Optimized process flowsheet," is produced.

As mentioned in Section 1.3, different non-ideal cases of project execution are represented in the iteration loops, e.g., a case when more experimental resources are requested from a design stage. Another particular non-ideal case in process design is the gap between opinions of process chemists and process engineers. For instance, specifications on reaction chemistry considered only by reaction chemists can serve as a severe constraint on designing separation processes. To avoid such a situation, the model shown in Figure 6 defines a direct iteration loop of "Feedback from process engineers" between activities A3 and A4, as a desired exchange of information between chemists and engineers.

As an example of work-step activities, the decomposition of Activity A3: Perform Process Chemistry II is presented in Figure 7. According to the "Selected routes" in Process Chemistry I (activity A2), experiments are performed in activity A32, which provide "Reaction information" including reaction performance (conversion, selectivity) and conditions (temperature, pressure). On the basis of this information, models for reaction alternatives are created in activity $\mathrm{A} 33$. The outcome, "Mass and energy balances", is the basis in activity A34: Make process evaluation performed. Here, the reaction routes are evaluated according to multiobjective criteria of the framework: economy, lifecycle environmental impacts and EHS hazard, together with technical aspects as a constraint. Finally, all evaluation results are summarized and multiobjective decision-making (Sugiyama et al., 2008) is performed in activity A35: Select reaction route(s). Similar to the main-level activities in Figure 6, feedback paths are connected to activity A31 from the other activities, which are activated in a non-ideal situation. The subactivities of A2, A4 and A5 can be described by the same manner. In activities for multiobjective decisionmaking at four design stages, the relevant component of the control arrow is different. 


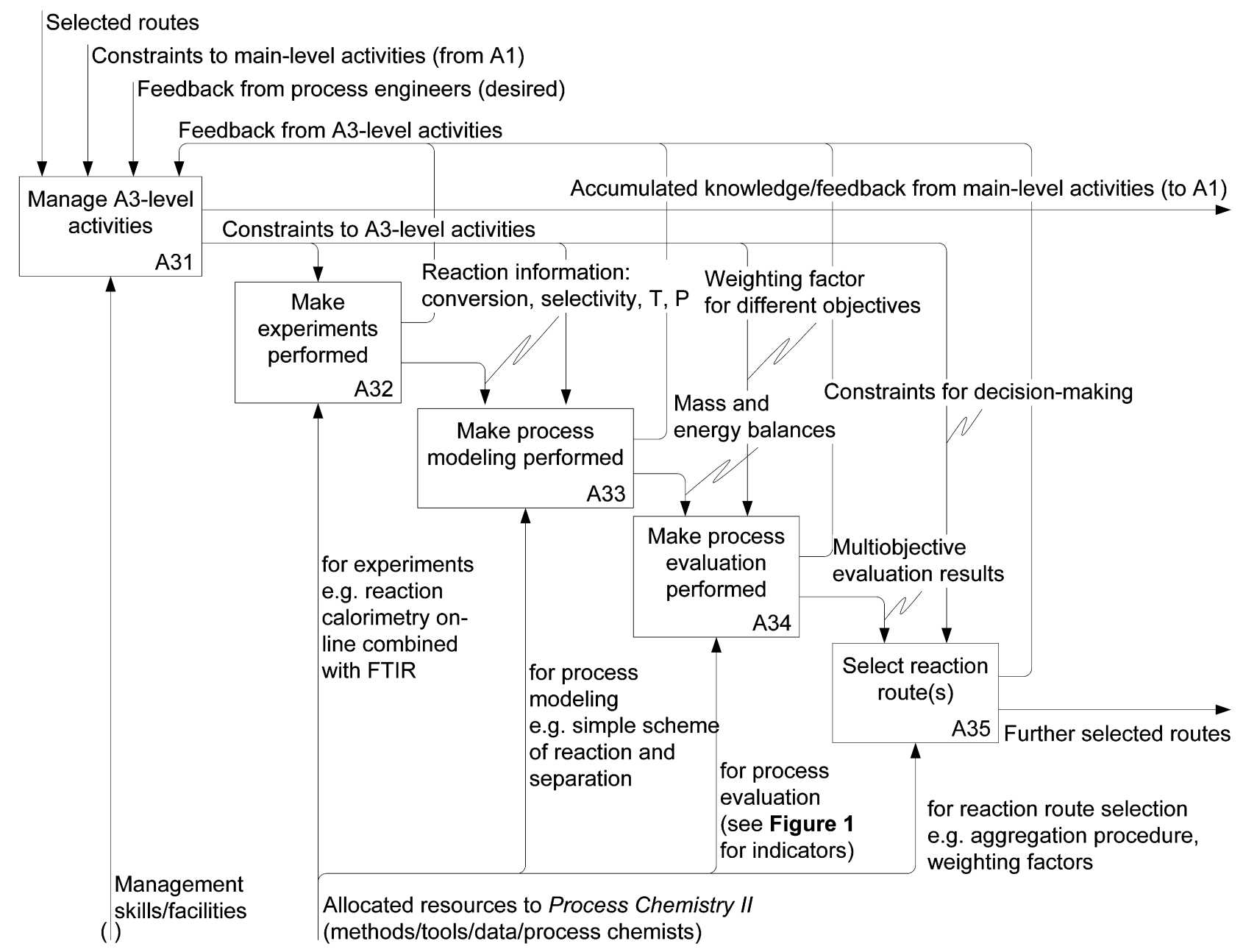

Fig. 7 Decomposition of activity A3: Perform Process Chemistry II as example of work-step activities, i.e. A2 to A5, in Figure 6

2.2 Impact of design constraints on decision-making characterized by MMA process development

Generally different design constraints influence decisions at different design stages. In Figure 6, each of the control arrows from outside to activity A1 has different impacts on the output arrows produced at activities A2 to A5.

To characterize different impacts of the design constraints on different decisions, we investigated on the actual methacrylate (MMA) process development in Japan and in Europe. Today various processes exist to produce MMA using different reaction routes. The most traditional and major MMA process is so-called acetone cyanohydrin $(\mathrm{ACH})$ process, which was launched in the 1930s in Europe. In this process, ACH is first synthesized from acetone and $\mathrm{HCN}$, and is further esterified to MMA using $\mathrm{H}_{2} \mathrm{SO}_{4}$. As a coupled byproduct, ammonium bisulfate is produced, which is oxidized to recover $\mathrm{H}_{2} \mathrm{SO}_{4}$. Since its commercialization, the ACH process was the only industrial MMA process worldwide for a long time. In the 1970s/80s, several Japanese MMA producers developed alternative processes, using oxidation of C4-remainings such as isobutene or tert-butyl alcohol (TBA). To date, the EU and US market of MMA is highly dominated by the $\mathrm{ACH}$ process, while the Japanese market is shared by the $\mathrm{ACH}$ and $\mathrm{C} 4$-based processes. By interviewing Japanese and European MMA producers, we analyzed how they decided differently in the 1970s/80s and characterized how different design constraints influence on decision-making at different stages.

"Prior decisions on the process" have a large impact on decisions both at Process Chemistry and Conceptual Design stages. The production scale requires proper selection on reaction chemistry, e.g., raw materials with sufficient availability and catalyst with sufficient activity, and on process technology, e.g., separation agents and waste treatment methods. Product quality can always be critical. This was the case in MMA processes where high transparency is demanded from the customer. At the level of Process Chemistry, reaction byproducts were investigated whether they remain as color impurities; while at the level of 


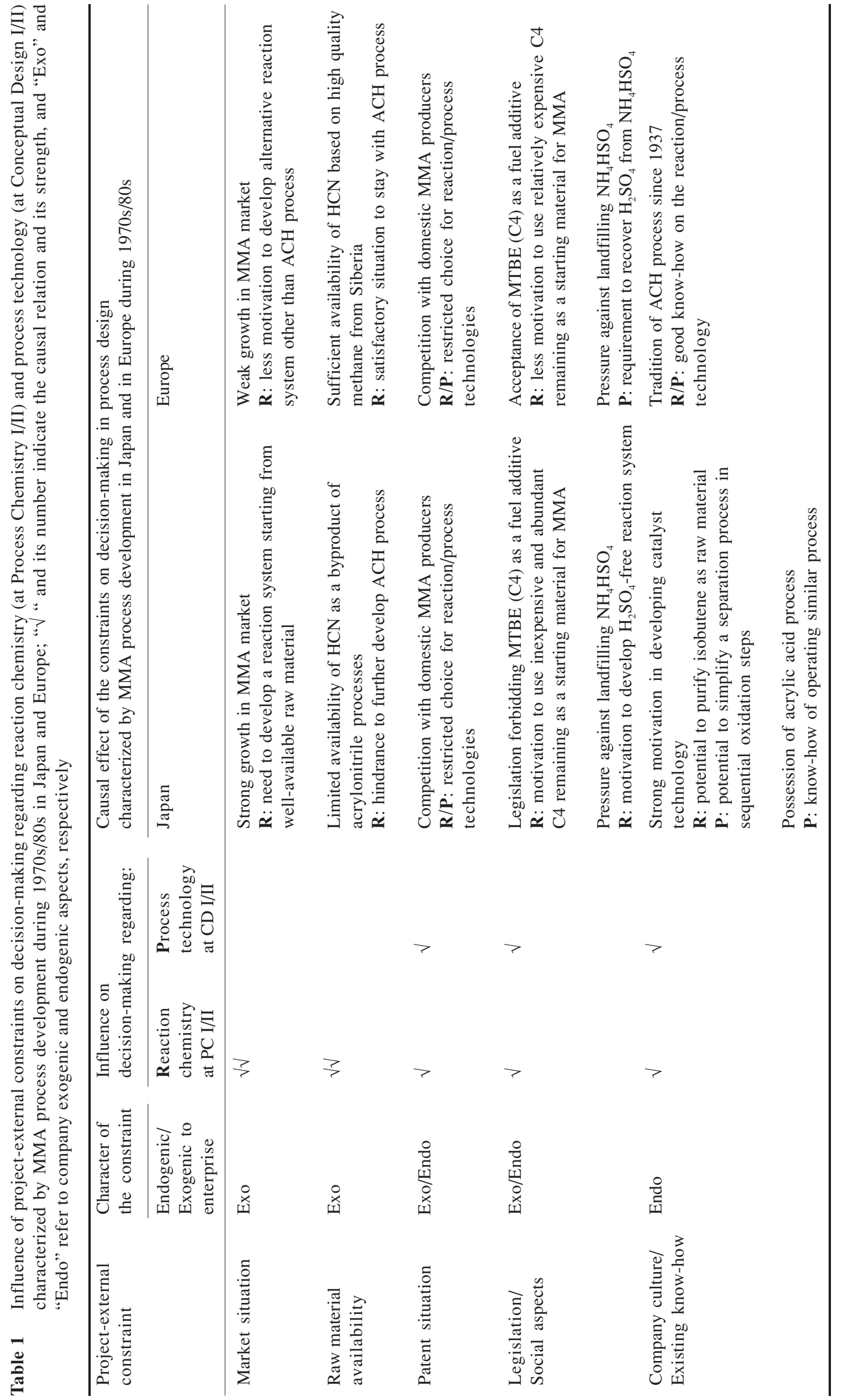




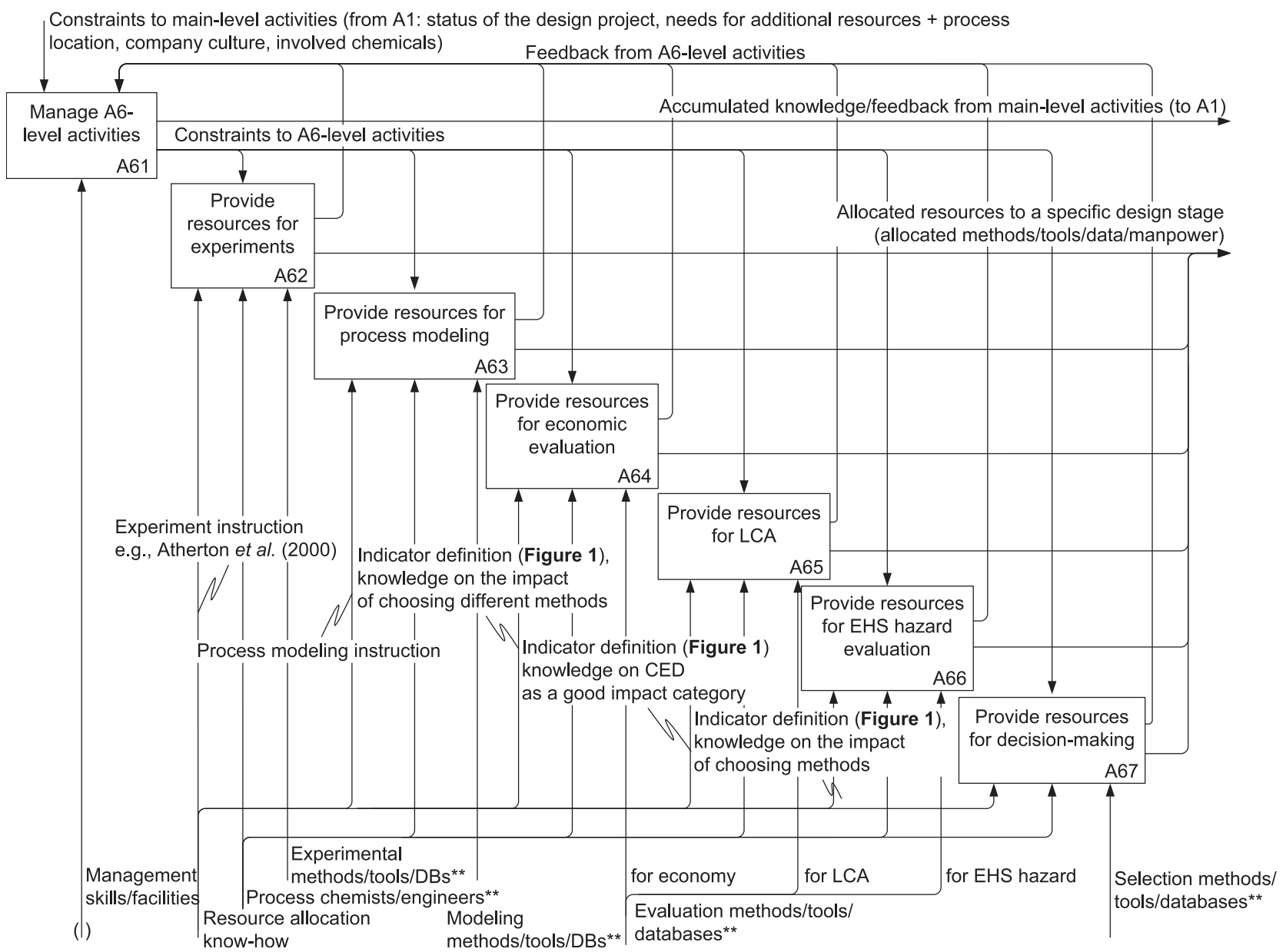

Fig. 8 Decomposition of Activity A6: Provide resources in Figure 6; ** indicates the status of resources as "available but unallocated" (compare with Figure 6)

Conceptual Design, purification processes were examined whether the final product quality meets the desired transparency. Different locations may also change the decisions in both reaction chemistry and process technology together with the other constraints, e.g., market situation in the region.

The remaining constraints are characterized in Table 1. Impacts of control arrows from "Market situation" to "Company culture/Existing process" in Figure 6 on different decisions are investigated. The basis of this semi-quantification is the MMA process situation in Japan and Europe during the 1970s/80s, where each design constraint had different causal effects in the two regions. "Market situation" and "Raw material availability" are classified as constraints with strong impacts on decisions at Process Chemistry I/II, particularly with regard to synthesis routes. In the MMA case, Japanese producers needed to develop alternative processes which start from sufficiently available $\mathrm{C} 4$ materials to meet the rapidly growing demand. European producers could stay with the traditional $\mathrm{ACH}$ process, which can deal with a weakly growing market by debottlenecking. "Patent situation" can be relevant at all design stages. In the MMA case, producers made more effort in domestic competition at that time; thus, there was no effective blockage of patents between the two regions. "Legislation/Social aspects" is another constraint that can influence decisions at all stages. For MMA reaction chemistry, different levels of regional legislation on MTBE, which is a typical transportation form of $\mathrm{C} 4$ remaining, resulted in different selections of reaction routes. Pressure against ammonium bisulfate, an inherent byproduct from the $\mathrm{ACH}$ process, triggered the motivation to develop a $\mathrm{H}_{2} \mathrm{SO}_{4}$-free MMA chemistry for Japanese producers, or the requirement to properly treat the waste acid for European producers. The last and sole enterprise-endogenic constraint "Company culture/Existing knowhow" is also important at all design stages. For Japanese MMA producers, there was a high potential to develop new catalytic technologies; this resulted in the development of alternative C4-based processes that required a breakthrough in catalysts. Existing processes used by Japanese producers, e.g., the acrylic acid process, supported the development of similar C4-based processes, where know-how of separation, purification 
and control strategy can be easily applied. For European MMA producers, the long tradition of the $\mathrm{ACH}$ process was a good reason for continued use of this established technology.

Table 1 shows that there are more external design constraints relevant at Process Chemistry I/II than at Conceptual Design I/II. This observation indicates that the design-project manager should pay more attention to project-external constraints at Process Chemistry stages. At Conceptual Design stages, decisions taken at prior design stages might serve as project-internal constraints such as reaction routes, solvents, and catalyst. For decision-making at these stages, the manager should incorporate both project-internal and -external constraints equally in a balanced manner. The above knowledge is an important part of the mechanism arrow "Management skills/facilities" that the designproject manager exploits in activity A1.

\subsection{Activities providing resources for different process design stages}

Figure 8 shows the decomposition of activity A6: Provide resources in Figure 6. At different design stages, the design-project manager provides "Process chemists/engineers" and "Methods/tools/databases" for experiments (activity A62), modeling (A63), and evaluation with respect to economy (A64), life-cycle environmental impacts (A65), EHS hazard (A66), and decision-making (A67). Compared with the traditional process design, activities related to non-monetary issues and multiobjective decision-making, i.e. activities A65 to A67 are newly required. Here, "Resource allocation know-how" plays a crucial role in providing appropriate resources. The most important component of this mechanism is the overview of the design framework shown in Figure 1, which is a guideline for providing modeling and evaluation methods at different design stages. Another component is knowledge of the impact of selecting process options and evaluation settings on the assessment result. For instance, Guntern (1999) and Sugiyama (2007) quantified the significant impact of determining reaction chemistry as compared with specifying process structure and parameters. Koller et al. (2001) and Adu et al. (2008) also presented large changes on the assessment results caused by applying different EHS hazard evaluation methods. This knowledge demands the designproject manager to carefully allocate proper resources to design stages, especially at early Process Chemistry stages.

The sub-activities of A62 to A67 consist of elements of the template in Figure 4(b), and as an example, activity A65: Provide resources for LCA is presented in detail in Figure 9. Through the control arrow from activity A61, the current stage of the design is notified to activity A651, which triggers activity A652: Prefix evaluation indicator. In this activity, the corresponding indicator for evaluating life-cycle en- vironmental impacts, e.g., raw material CED for Process Chemistry I/II, is prefixed by referring to Figure 1 as "Resource allocation know-how". Here, "Knowledge on CED as a good impact category" based on Huijbregts et al. (2006) supports the application of CED; however, the appropriateness of this choice is examined in activity A653: Study feasibility of CED as an impact category. A helpful analysis here is to check the correlation between CED and full-LCA score, e.g., Eco-indicator 99 point (EI99: Goedkoop and Spriensma, 1999), of "Involved chemicals" at the current stage. The content of "Involved chemicals" is updated along with the design stage, e.g., by introducing separation agents when the design starts to cover the separation part at Conceptual Design stages. Thus, whenever the design proceeds from one stage to the next, activity A653 should be performed again. The other output, "Pre-allocated resources for LCA," includes data values, tools and manpower that were applied in activity A653. Receiving the result of the feasibility study, the project manager performs activity A654: Fix evaluation indicator, and according to its output "Fixed evaluation indicator", activity A655: Finalize resource allocation for LCA is executed. "Preallocated resources for LCA", which were produced in activity A653, are transformed to the final form of "Allocated resources for LCA to a specific design stage." As the design stage proceeds, the character of LCA data becomes more specific and detailed, i.e. Process Chemistry stages require only CED values of raw materials, while Conceptual Design stages demand, in addition, separation agents and energy utility.

Similarly, the feasibility of pre-selected methods of EHS hazard evaluation is investigated in activity A663 within activity A66: Provide resources for EHS hazard evaluation. For this validation, the manager checks whether the pre-selected method is appropriate for the design contest. In particular, matching to "Process location" and "Company culture/Existing know-how," included in the control arrow and originated from the project-external constraints (see Figure 6), are important. For instance, when designing a process at a sea shore, the hazard evaluation method should cover water hazards in a relevant way; when selecting a method, considered parameters and mathematical formulation for hazard calculation should fit to the policy of the design manager and the personnel who execute the evaluation.

These mechanism-providing activities are open for new design criteria. For instance, design uncertainty such as future change of the business, including the variation of process utilization rate and technology situation, plays a crucial role in the actual cases. Incorporation of such mechanisms in the design, e.g., by presuming risk-distribution for uncertain factors, can be discussed in a similar manner that EHS mechanisms are included. 


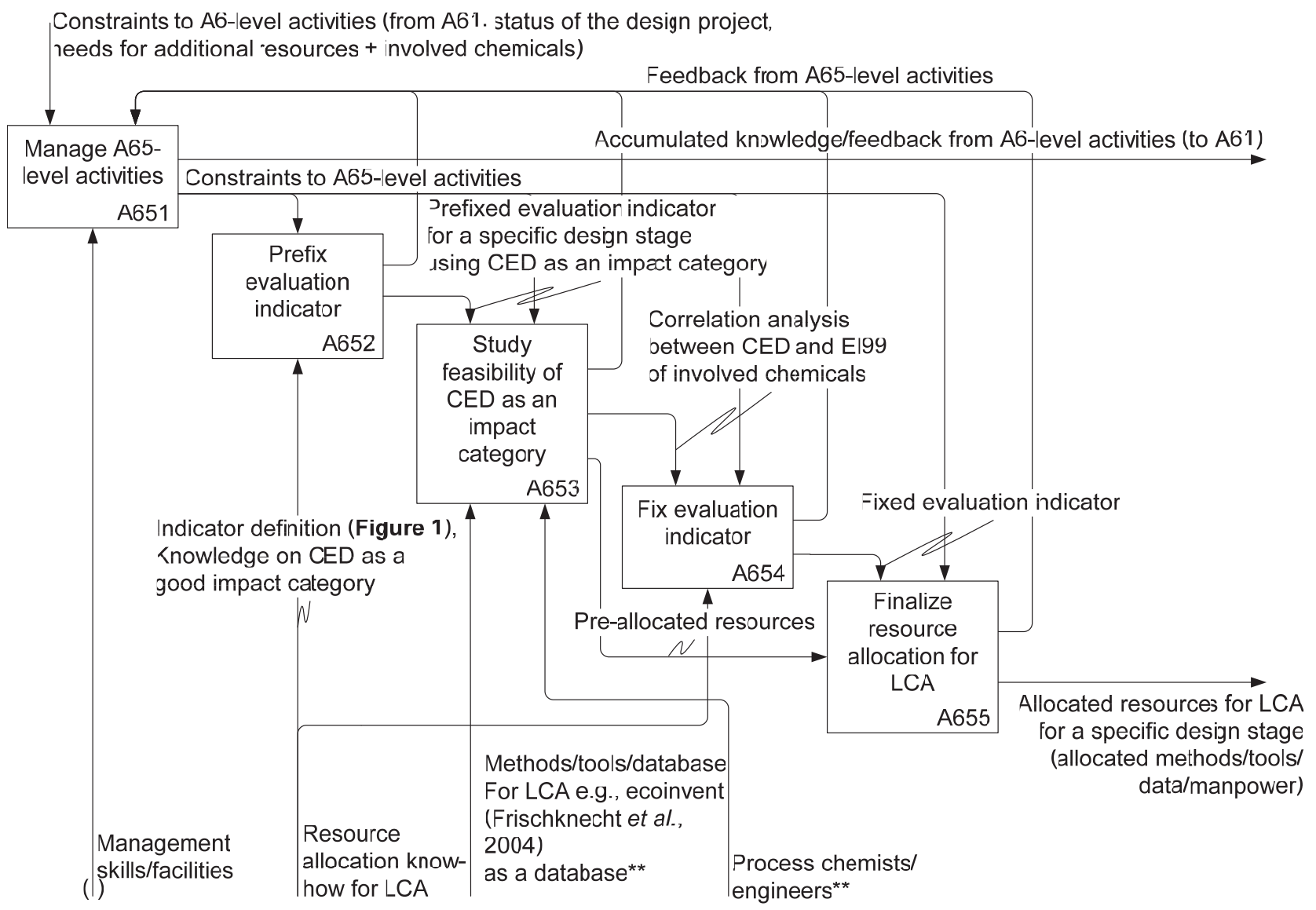

Fig. 9 Decomposition of activity A65: Provide resources for LCA in Figure 8 based on template in Figure 3; ** indicates the status of resources as "available but unallocated" (compare with Figure 6); EI99 refers to Eco-indicator 99 by Goedkoop and Spriensma (1999)

\subsection{Summary of important know-how for design- project manager}

Table 2 summarizes the changing character of decision-making, and mechanisms over stages of Process Chemistry I/II and Conceptual Design I/II, which are observed in previous sections. Specifying reaction chemistry has the character of a big decision with a large influence at the late stages, while selecting process technology involves fine-tuning and optimization. Regarding constraints, project-external and especially enterprise-exogenic ones are relevant at Process Chemistry stages, while at Conceptual Design stages, both project-external and -internal ones are important. The mechanisms at the earlier two stages are rather general and wide-scoped, which evolve to be more specifically focused at the later stages.

On the basis of this general summary, the following findings are drawn. For making influential decisions at Process Chemistry stages, the design-project manager should consider a broad range of project-external constraints and provide wide-scoped resources. For fine-tuning-type decisions at Conceptual Design stages, the manager should look for various constraints inside and outside the project, and allocate elaborate mechanisms for specific purposes. Here, decisions on reaction chemistry at previous Process Chemistry stages should not excessively restrict the choice of process technology. Thus, between two phases, the manager should offer opportunities of communication between chemists and engineers through the direct iteration-loop from activity A4 to A3. These findings are an important part of the know-how of the designproject manager, which are represented as the mechanisms "Management skills" and "Resource allocation know-how" in the activity model shown in Figure 6.

\section{Conclusions and Outlook}

We have presented activity models of chemical process design integrating EHS evaluation as a new element with conventional economic and technical considerations. A design framework, defines four stages of early process design, i.e. Process Chemistry I/II and Conceptual Design I/II, with appropriate indicators for 
Table 2 Changing characters of decision-making, constraints and mechanisms in Process Chemistry I/ II and Conceptual Design I/II

\begin{tabular}{lll}
\hline Character of: & at Process Chemistry I/II & at Conceptual Design I/II \\
\hline Decision-making & Highly influential & Fine tuning and optimization \\
& - Reaction route selection & - Selection of separation sequence \\
& - Reaction recipe specification & - Equipment sizing \\
Constraint & Mainly project-external & Both project-external and -internal \\
& - Prior decisions on the process & - Prior decisions on the process \\
& - Market situation & (scale, location, quality) \\
& - Natural resources & - Company culture/Existing know-how \\
& - Patent situation & - Legislation/Social aspects \\
& - Legislation/Social aspects & - Patent situation \\
& & - Selected reaction routes, solvent, etc in \\
Mechanism & General/Wide scoped & Process Chemistry I/II \\
& e.g. & Specific/Narrow focused \\
& - Raw material prices & e.g. \\
& - CED of raw materials & - Equipment prices \\
& - Flash point of solvents, auxiliaries & - Flash point of extractive agents \\
\hline
\end{tabular}

multiobjective evaluation. IDEF0 was selected as an activity modeling method for hierarchical and transparent description of complex design activities. A set of template models was created, which describe how a manager executes a project step-by-step while exploiting resources and handling constraints appropriately. This general template model functioned as a format basis of translating the design framework as IDEF0 models.

According to the template, the created activity model described a way a design-project manager makes four design stages performed while providing constraints and resources appropriately to different stages. On the basis of the traditional design procedure, EHSrelated activities, tools and information are defined as new elements of process design. The application of IDEF0 enabled the systematic definition of the design activity at different levels of detail, from the top level to the sub-activity levels. Using this activity model, two detailed analyses were performed from the sides of control and mechanism. In one analysis, the impact of different design constraints, e.g., market situation, competitors patents, or company culture, on decisionmaking at different design stages, was investigated. The actual development story of MMA processes in Japan and in Europe during the 1970s/80s characterized constraints considered in the activity model. In another detailed analysis, activity in which the design manager provides resources for design stages are decomposed into sub-activities, i.e. activities that allocate methods and data for environmental LCA. This detailed analysis highlighted newly required activities, information, tools and managerial know-how for incorporating nonconventional EHS evaluation of a design alternative.
In the paper, we finally summarized the findings obtained from the above analyses as important know-how for the design-project manager in executing the integrated design framework.

IDEF0 activity modeling was proven to be a powerful tool for incorporating EHS consideration as a new element in the already-existing and highly-complicated business of process design. Towards the realization of sustainable process development, activity modeling offers a key means of systematically integrating wellbalanced triads of economy, ecology and society.

\section{Acknowledgements}

The authors are grateful to the experts of Degussa and Sumitomo Chemicals. Many thanks go to Mr. Yasunori Kikuchi of the University of Tokyo for his contribution. Financial support from Nagai Foundation Tokyo is greatfully acknowledged. Part of this work was financially supported by a Grant-in-Aid for Scientific Research (B) No. 18310052, and Alliance for Global Sustainability.

\section{Literature Cited}

Adu, I. K., H. Sugiyama, U. Fischer and K. Hungerbühler; "Comparison of Methods for Assessing Environmental, Health and Safety (EHS) Hazards in Early Phases of Chemical Process Design," Process Saf. Environ. Prot., 86, 77-93 (2008)

Azapagic, A. and R. Clift; "Linear Programming as a Tool in Life Cycle Assessment," Int. J. LCA., 3, 305-316 (1998)

Cano-Ruiz, J. A.; Decision Support Tools for Environmentally Conscious Chemical Process Design, Ph.D Thesis, MIT, U.S.A. (2000)

Cano-Ruiz, J. A. and G. J. McRae; "Environmentally Conscious Process Design," Annu. Rev. Energy Env., 23, 499-536 (1998)

Chen, H. and D. R. Shonnard; "Systematic Framework for Environmental Conscious Chemical Process Design: Early and Detailed Design Stages," Ind. Eng. Chem. Res., 43, 525-552 (2004)

Fuchino, T. and Y. Shimada; "IDEF0 Activity Model Based Design Rationale Supporting Environment for Lifecycle Safety," Lecture Notes in Artificial Intelligence, 2773, 1281-1288 (2003) 
Fuchino, T., T. Wada and M. Hirao; "Acquisition of Engineering Knowledge on Design of Industrial Cleaning System through IDEF0 Activity Model," Lecture Notes in Artificial Intelligence, 3214, 418-424 (2004)

Gabber, H. A., A. Aoyama and Y. Naka; "Model-Based ComputerAided Design Environment for Operational Design," Comput. Ind. Eng., 46, 413-430 (2004)

Goedkoop, M. and R. Spriensma; The Eco-indicator 99: A Damage Oriented Method for Life Cycle Impact Assessment, Methodology Report, PRé Consultants, Amersfoort, the Netherlands (1999)

Guntern, C.; Ecoefficiency as a Guideline for Chemical Process Development (in Germany), Dissertation ETH No. 13029, ETH Zurich, Zurich, Switzerland (1999)

Heinzle, E., D. Weirich, F. Brogli, V. H. Hoffmann, G. Koller, M. A Verduyn and K. Hungerbühler; "Ecological and Economic Objective Functions for Screening in Integrated Development of Fine Chemical Process. 1. Flexible and Expandable Framework Using Indices," Ind. Eng. Chem. Res., 37, 3395-3407 (1998)

Hilaly, A. K. and S. K. Sikdar; "Pollution Balance Method and the Demonstration of Its Application to Minimizing Waste in a Biochemical Process," Ind. Eng. Chem. Res., 34, 2051-2059 (1995)

Hoffmann, V. H., K. Hungerbühler and G. J. McRae; "Multiobjective Screening and Evaluation of Chemical Process Technologies," Ind. Eng. Chem. Res., 40, 4513-4524 (2001)

Huijbregts, M. A. J., L. J. A. Rombouts, S. Hellweg, R. Frischknecht, A. J. Hendriks, D. van de Meent, A. M. J. Ragas, L. Reijnders and J. Struijs; "Is Cumulative Fossil Energy Demand a Useful Indicator for the Environmental Performance of Products?," Environ. Sci. Technol., 40, 641-648 (2006)

ISO 14040, Environmental Management_Life Cycle AssessmentPrinciples and Framework (1997)

ISO 14041, Environmental Management-Life Cycle AssessmentGoal and Scope Definition and Inventory Analysis (1998)

ISO 14042, Environmental Management_Life Cycle AssessmentLife Cycle Impact Assessment (2000)

ISO 14043, Environmental Management-Life Cycle AssessmentLife Cycle Interpretation (2000)

Kheawhom, S. and M. Hirao; "Environmentally Benign Separation Process Synthesis," J. Chem. Eng. Japan, 37, 243-252 (2004a)

Kheawhom, S. and M. Hirao; "Decision Support Tools for Environmentally Benign Process Design under Uncertainty," Comput. Chem. Eng., 28/29, 1715-1723 (2004b)

Kikuchi, Y. and M. Hirao; "Integrated Design of Process and Operation Considering Local Risks and Global Impacts: A Case Study on Metal-Degreasing Process Design," 17th European Symp. on Computer Aided Process Engineering, Vol. 24, pp. 1223-1228, Elsevier, Amsterdam, the Netherlands (2007)

Killich, S., H. Luczak, C. Schlick, M. Weissenbach, S. Wiedenmaier and J. Ziegler; "Task Modelling for Cooperative Work," Behav. Info. Technol., 18, 325-338 (1999)
Kniel, G. E., K. Delmarco and J. G. Petrie; "Life Cycle Assessment Applied to Process Design: Environmental and Economic Analysis and Optimization of a Nitric Acid Plant," Environ. Progress, 15, 211-228 (1996)

Koller, G., U. Fischer and K. Hungerbühler; "Assessing Safety, Health, and Environmental Impact during Early Process Development," Ind. Eng. Chem. Res., 39, 960-972 (2000)

Koller, G., U. Fischer and K. Hungerbühler; "Comparison of Methods Suitable for Assessing the Hazard Potential of Chemical Processes during Early Design Phases," Process Saf. Environ. Prot., 79, 157-166 (2001)

Naka, Y.; Introduction to Integration Engineering (Togogaku Nyumon), Kogyo Chosakai, Tokyo, Japan (2006)

National Institute of Standards and Technology (NIST); Integration Definition for Function Modeling (IDEF0), Federal Information Processing Standards Publication No. 183, Department of Commerce, Gaithersburg, U.S.A. (1993)

PIEBASE; Process Industries Executive for Achieving Business Advantage Using Standards for Data Exchange, http:// www.posc.org/, Houston, U.S.A. (2007)

Ross, D. T.; "Structured Analysis (SA): A Language for Communication Ideas," IEEE Trans. Software Eng., 3, 16-35 (1977)

Ross, D. T.; "Application and Extensions of SADT," Computer, 18, 25-34 (1985)

Ross, D. T. and K. E. Schoman; "Structured Analysis for Requirements Definition," IEEE Trans. Software Eng., 3, 6-15 (1977)

Schneider, R. and W. Marquardt; "Information Technology Support in the Chemical Process Design Life Cycle," Chem. Eng. Sci., 57, 1763-1792 (2002)

Saling, P., A. Kicherer, B. Dittrich-Krämer, R. Wittlinger, W. Zombik, I. Schmidt, W. Schrott and S. Schmidt; "Eco-Efficiency Analysis by BASF: The Method," Int. J. LCA., 7, 203-218 (2002)

Sugiyama, H.; Decision-Making Framework for Chemical Process Design Including Different Stages of Environmental, Health and Safety (EHS) Assessment, Dissertation ETH No. 17186, http://e-collection.ethbib.ethz.ch/show?type=diss\&nr=17186, ETH Zurich, Zurich, Switzerland (2007)

Sugiyama, H., M. Hirao, R. Mendivil, U. Fischer and K. Hungerbühler; "A Hierarchical Activity Model of Chemical Process Design Based on Life Cycle Assessment," Process Saf. Environ. Prot., 84, 63-74 (2006)

Sugiyama, H., U. Fischer, M. Hirao and K. Hungerbühler; "Decision Framework for Chemical Process Design Including Different Stages of Environmental, Health, and Safety Assessment," AIChE J., 54, 1037-1053 (2008)

U.S. Air Force; Air Force Computer Aided Manufacturing (AFCAM) Master Plan, AFML-TR-74-104, Air Force Base, WrightPatterson, U.S.A. (1974)

Verein Deutscher Ingenieure; VDI-Richtlinie 4600: Cumulative Energy Demand, Terms, Definitions, Methods of Calculation, Verein Deutscher Ingenieure, Düsseldorf, Germany (1997) 